\title{
Design and Implementation of a Multi-Standard Event-Driven Energy Management System For Smart Buildings
}

\author{
Iman Khajenasiri*, Edoardo Patti ${ }^{\uparrow}$, Marco Jahn ${ }^{\S}$, Andrea Acquaviva $₫$, Marian Verhelst* \\ Enrico Macii ${ }^{\Uparrow}$, Georges Gielen* \\ *Dept. of Electrical Engineering-ESAT, MICAS, KU Leuven, Belgium. \\ IDept. of Control and Computer Engineering, Politecnico di Torino, Italy.

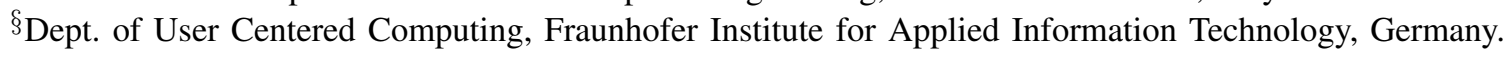

\begin{abstract}
This paper presents the design and implementation of a multi-standard energy management system, which leverages heterogeneous devices to convert existing buildings into Smart Buildings. Its main purpose is to increase the energy efficiency of buildings providing user awareness to promote green behaviors. The proposed solution has been designed to enable interoperability across different standards and protocols in order to develop applications with which end users can interact with the system. Finally, a web portal and a smartphone application to give feedback and to view environmental information are presented.
\end{abstract}

\section{INTRODUCTION}

Nowadays, the number of energy-consuming appliances in public buildings has increased drastically. This increase demands for energy management systems to reduce the energy consumption. A full implemented system requires a welldeveloped hardware infrastructure, a middleware and its supporting software with an interactive interface for the user [1]. A smart-building energy system should be able to provide a low-latency and user-friendly control system for managing versatile appliances in a building. The user's configurations and commands should be supervised in a friendly manner at a high level in which the energy analysis results are abstracted and displayed. Moreover, the emerging Internet of Things (IoT) market necessitates the development of an effective web-service interface between users, distributed applications and the environmental heterogeneous technologies. A comprehensive solution should perform a hardware to application layer implementation for user-centric applications. In this work, we present the design and implementation of a smart energy management system that can be applied to existing buildings, providing an intelligent ICT-based monitoring and managing system. The proposed platform includes the software infrastructure, the deployed heterogeneous sensor networks, an integration layer and the web-service user interface.

\section{HARDWARE INFRASTRUCTURE}

In this work, both existing off-the-shelf commercial wireless devices and a prototype low-energy UWB transceiver have been taken into account. They exploit different communication protocols such as IEEE 802.15.4, ZigBee, EnOceans and UWB. The purpose of this heterogeneous integration (see Section III) is to increase the autonomy and scalability of the system as well as the adaptation to emerging technologies.
More specifically, four different devices have been integrated. The TelosB sensor node communicates with the IEEE 802.15.4 protocol and is equipped with light, temperature and humidity sensors. A prototype board built on Texas Instruments' CC2530 exploits the ZigBee protocol to send information about temperature and illuminance. The ST SmartPlug adopts the ZigBee protocol to control the appliance connected to it and to measure its power consumption. The STM300 sensor node exploits the Enocean protocol, which works in the $868 \mathrm{MHz}$ UHF band, and sends data about motion, temperature and illuminance. The aforementioned sensor nodes have a high energy consumption because of the use of energy-hungry protocols such as ZigBee. Therefore, in addition, a prototype version of a fully integrated custom-designed pulsed-UltraWideband (UWB) transceiver is implemented as an ultralow-energy device. It provides a lower energy-per-bit value compared to existing products. The specifications of the end nodes are shown in Table I. The custom-designed UWB technology has a ten times better energy efficiency compared to the commercial wireless technologies for smart-building applications [4]. The UWB sensor has also been integrated in the whole software infrastructure to be presented in Section III.

TABLE I. SPECIFICATIONS OF THE APPLIED WIRELESS SENSOR AND ACTUATOR NODES

\begin{tabular}{|l|l|l|l|l|l|}
\hline $\begin{array}{l}\text { Sensor } \\
\text { node } \\
\text { product }\end{array}$ & STM300 & CC2530 & TelosB & $\begin{array}{l}\text { ST } \\
\text { Smart- } \\
\text { Plug }\end{array}$ & UWB \\
\hline \hline $\begin{array}{l}\text { Radio } \\
\text { Technol- } \\
\text { ogy }\end{array}$ & EnOcean & ZigBee & $\begin{array}{l}\text { IEEE } \\
802.15 .4\end{array}$ & ZigBee & UWB \\
\hline $\begin{array}{l}\text { Energy } \\
\text { consump- } \\
\text { tion }\end{array}$ & $403 \mathrm{~nJ} / \mathrm{bit}$ & $348 \mathrm{~nJ} / \mathrm{bit}$ & $69.6 \mathrm{~nJ} / \mathrm{bit}$ & $781 \mathrm{~nJ} / \mathrm{bit}$ & $5.31 \mathrm{~nJ} / \mathrm{bit}$ \\
\hline $\begin{array}{l}\text { Measured } \\
\text { parameter }\end{array}$ & $\begin{array}{l}\text { Motion } \\
\text { Temperature } \\
\text { Illuminance }\end{array}$ & $\begin{array}{l}\text { Temperature } \\
\text { Illuminance }\end{array}$ & $\begin{array}{l}\text { Temperature } \\
\text { Humidity } \\
\text { Illuminance }\end{array}$ & $\begin{array}{l}\text { Power } \\
\text { Energy }\end{array}$ & Temperature \\
\hline
\end{tabular}

\section{SOFTWARE INFRASTRUCTURE}

In a Smart-building context, where heterogeneous sensor nodes are already deployed or to be installed, the interoperability between them is a big issue. The software infrastructure, presented in this section, exploits a web-serviceoriented approach in order to manage the heterogeneous devices based on different protocols, enabling the interoperability 
and providing hardware abstraction. The starting point for this work was the LinkSmart middleware [3]. It provides features for enabling a peer-to-peer (P2P) communication, based on events, directing connect all the devices inside the LinkSmart network, no matter if they are behind a firewall or the Network Address Translator. The event-based communication exploits the publish/subscribe approach for LinkSmart web services, which allows the development of loosely coupled event-based applications. As shown in Figure 1, the proposed solution is a three-layered software infrastructure with i) The Integration Layer, ii) The Middleware Layer and iii) The Application Layer.

The Integration Layer, which is the lowest layer, is in charge of enabling the interoperability across heterogeneous devices with a dedicated interface called proxy. It acts as a bridge between the LinkSmart network and the underlying technology, so a specific proxy needs to be developed for each technology. This modular approach, based on proxies, provides to the whole infrastructure flexibility with respect to new emerging standards and technologies. At this layer, each device can be accessed by the middleware's upper layers to get data or to send commands. Moreover, the data collected realtime by the nodes are immediately sent to the middleware thanks to the Event Manager, which implements the eventbased communication. In this work, the heterogeneity and interoperability issues have been proven by applying IEEE 802.15.4, ZigBee, UWB and EnOcean wireless sensor nodes in the same system.

The Middleware Layer enables the P2P communication and provides the event-based approach. In addition, a specific component has been developed to give additional information about the building and its occupancy, which are useful to develop rules and control strategies to minimize the energy waste [2].

The Application Layer represents the higher layer: it provides a set of API's for developing distributed event-based applications to manage the building and to provide feedback to end users. In the implemented system three applications have been developed: i) a HVAC control system [2], ii) a smartphone application and iii) a web portal.

Finally, after utilizing the hardware and software in a pilot campus, an average energy saving of $71 \%$ is achieved for three sample days during summertime [2].

\section{WEB PORTAL AND SMARTPHONE APPLICATION}

In order to access the information coming from the building and to provide user awareness, a web portal and a smartphone application have been developed (Figure 2). Their purpose is to show feedback to end users on the energy consumption, encouraging energy savings by displaying the energy consumption measured by the system. Hence, in addition to the main functions for interfacing, configuring and controlling the devices, they also present an energy management analysis [5]. Indeed, the user can access to: i) real-time environmental data; ii) the occupancy status; iii) a temperature and energy comparison chart of rooms; iv) a list of devices connected to smart plugs per room; v) the energy consumption of smart plugs connected appliances; vi) the daily, monthly and yearly view and historic environmental values and occupancy charts. Three kinds of users have been identified for access to information:

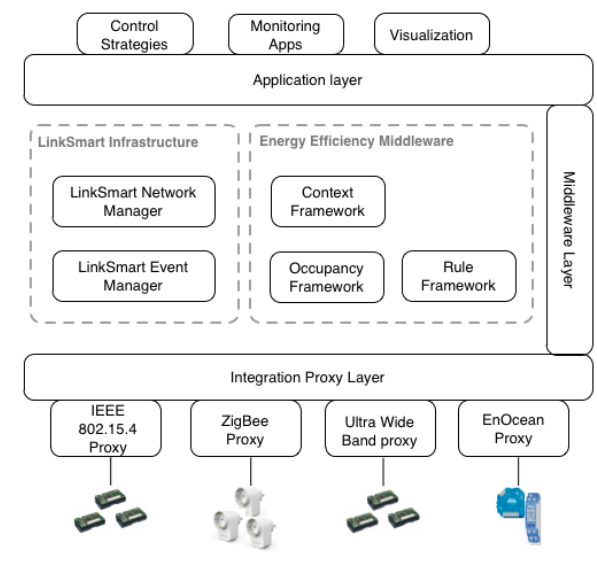

Fig. 1. Software energy management system infrastructure of the developed smart-home

- $\quad$ The Public User has limited access to the information of the rooms and may inspect only the public spaces;

- The Office Owner, after authentication, may examine information about his own office and the public spaces;

- The Technician, after authentication, has full access to the information about the entire building. Also in his private page, he can see warnings about any malfunction in the entire monitored building.

In addition the smartphone application shows this information exploiting both Virtual and Augmented Reality.

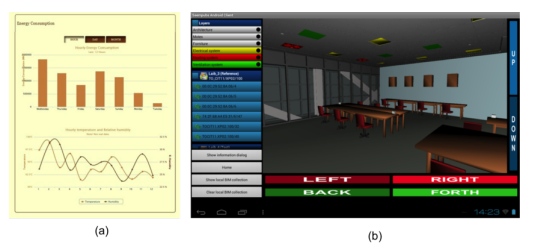

Fig. 2. Developed (a) web portal and (b) smartphone application

\section{CONCLUSION}

The design and implementation of a multi-standard energy management system for smart buildings has been presented. The proposed solution has classified general requirements of smart building energy management system from bottom-totop layers using heterogeneous devices for measuring different environmental parameters. For an applied pilot campus, an average energy saving of $71 \%$ has been obtained for sample days in summertime.

\section{REFERENCES}

[1] Dae-Man Han; Jae-Hyun Lim, "Design and implementation of smart home energy management systems based on zigbee", IEEE Transactions on Consumer Electronics, vol.56, no.3, pp.1417-1425, Aug. 2010.

[2] Patti, E.; Acquaviva, A.; Jahn, M.; Pramudianto, F.; Tomasi, R.; Rabourdin, D.; Virgone, J.; Macii, E., "Event-Driven User-Centric Middleware for Energy-Efficient Buildings and Public Spaces," IEEE Systems Journal, pp.10, Feb. 2014.

[3] Eisenhauer, M.; Rosengren, P.; Antolin, P., "A Development Platform for Integrating Wireless Devices and Sensors into Ambient Intelligence Systems," in Proc. 6th Annu. IEEE Commun. Soc. Conf. SECON Workshops, Jun. 2009.

[4] Khajenasiri I.; Zhu P. ; Verhelst M.; Gielen G., "Low Energy UWB Transceiver Implementation For Smart Home Energy Management," IEEE 18th International Symposium of Consumer Electronics, , ISCE, Jun. 2014.

[5] http://energyportal.cnet.se/seempubs/index.aspx 